\title{
http://dx.doi.org/10.12795/PH.1995.v10.i01.14
}

\section{TIEMPO RELATIVO EN GRIEGO ANTIGUO}

\section{Rafael Martínez Vázquez}

0. El trabajo que sigue es un estudio de la expresión en griego antiguo de nociones incluidas entre las comúnmente conocidas como de tiempo relativo ${ }^{1}$. Consta de cuatro partes. Precede una introducción en que se definen las categorías de aspecto, tiempo y tiempo relativo, definiciones que están en la base del método empleado, o, si se prefiere, conforman la hipótesis de trabajo sobre la que se desarrolla todo el estudio. Comienza el estudio propiamente dicho con la descripción de los usos verbales a los que algunos autores, especialmente STAHL, atribuyen valores de tiempo relativo. Se ensaya en esta parte una delimitación de los contextos en que se dan estos empleos, así como una clasificación de excepciones y factores que estorban la expresión de estas nociones. Sigue una tercera parte en que se pasa revista a algunas de las tesis que se han expresado respecto a este fenómeno. Primero se reseñará a quienes lo niegan; luego se reseñará a quienes lo aceptan, con especial atención en este caso a cómo lo explican en relación con el sistema gramatical del verbo griego en su conjunto. En cuarto y último lugar se propone una explicación de los hechos, basada en el sistema de relaciones opositivas, que constituye la tesis central del trabajo.

\subsection{DEFINICIÓN DE ASPECTO, TIEMPO, TIEMPO RELATIVO². La categoría} gramatical de aspecto suele definirse, en lo que respecta al contenido, como aquella que caracteriza el hecho relatado por el verbo en sí, con referencia a su estructura interna ${ }^{3}$, mas sin referencia explícita a la situación comunicativa o a otro hecho relatado por otro verbo. El tiempo se define a su vez como la categoría que caracteriza el hecho relatado con referencia a la situación comunicativa. Precisamente esta referencia a la situación discursiva y, en definitiva, al hablante mismo, lo caracteriza como categoría deíctica. Frente a estas categorías, la taxis u orden temporal, más conocida como tiempo relativo ${ }^{4}$, caracteriza un hecho relatado con referencia a otro hecho relatado y sin referencia al hecho discursivo. Con respecto a la taxis, un hecho puede ser anterior, simultáneo o posterior a otro hecho. Estas nociones de Anterioridad, Simultaneidad, Posterioridad respecto a un evento relatado en el contexto inmediato, se gramaticalizan de modo diverso en las distintas lenguas. Estas

\footnotetext{
${ }^{1}$ También orden temporal o taxis. Tengo intención de utilizar indistintamente las tres expresiones a lo largo del trabajo.

2 Sigo en general a JACOBSON 1984:314-315. En lenguas como el latín o el español se postula la existencia de formas verbales cuyo valor es el de caracterizar un hecho relatado con referencia a otro hecho relatado e indirectamente también con referencia a la situación discursiva. El fenómeno se define unas veces como concordancia de tiempos y otras veces, desde otra perspectiva, como categoría de temporalidad. Paso por alto esta cuestión porque valores tales no se postulan, en principio, para el verbo griego antiguo.

3 COMRIE 1976.

4 Anteriority en KURYLOWICZ 1964.
} 
categorías tienen un ámbito de operatividad restringido. Como toda categoría semántica, se realizan en contextos adecuados y sencillamente no se actualizan o aparecen "neutralizadas" en otros contextos. Así, por ejemplo, en predicaciones atemporales (refranes, máximas, sentencias) es bien sabido que aparecen formas temporales del verbo pero sin actualizar operador temporal alguno. Cuestión metodológica esencial en este trabajo es la consideración de que la delimitación del ámbito operativo de una categoría no es menos relevante que la señalización de sus relaciones paradigmáticas con otras categorías del mismo dominio semántico o la descripción de sus contenidos.

\section{DESCRIPCIÓN DE LOS HECHOS 5 . El orden temporal se expresa en griego mediante} los temas aspectuales de presente y aoristo y el tema temporal de futuro. El tema de aoristo expresa anterioridad, el tema de presente expresa simultaneidad y el tema de futuro expresa posterioridad respecto al contenido de la predicación dominante.

\subsection{PRIMERA DELIMITACIÓN DEL ÁMBITO. Las nociones de tiempo relativo se} expresan típicamente en determinadas posiciones de dependencia sintáctica y semántica ${ }^{6}$. Son características de la proposición subordinada: sólo en ellas se puede comprobar su presencia de forma sistemática, esto es, gramaticalizada. Dado que son nociones relativas a términos presentes en el texto, y la predicación independiente no requiere ningún texto ajeno a sí misma para constituir un enunciado completo, no pueden constituirse en operadores fijos de la predicación independiente. Sí pueden serlo de la predicación subordinada, pues ésta depende siempre de una predicación independiente a la que puede remitir las citadas nociones. Por lo dicho no es extraño que en la predicación independiente el verbo (junto con los adverbios y demás expresiones pertinentes) exprese sistemáticamente el tiempo y el aspecto, pero no el orden temporal.

De modo que el ámbito de operatividad de la categoría de tiempo relativo viene delimitado en primer lugar por el entorno de subordinación. Más específicamente, por el grupo formado por oraciones completivas, de relativo, algunas adverbiales (esp. temporales y causales) y algunas construcciones deinfinitivo y participio. Paso a dar una somera relación de los datos debidamente ejemplificados.

\subsubsection{ORACIONES COMPLETIVAS ${ }^{7}$. El tiempo relativo se expresa tanto en formas de} indicativo como en las de optativo oblicuo o de subordinación.

\footnotetext{
${ }^{5}$ Hace ya tiempo STAHL (1907:164 ss.) y más recientemente RIJKSBARON (1984) incluyen las nociones de orden temporal en su descripción de los valores del verbo griego. RUIJGH (1971) ha sostenido que los temas "temporales" del verbo griego expresan nociones que encajan mejor en lo que hemos definido como orden temporal, que en lo que hemos definido como aspecto. HETTRICH (1976) recoge numerosos ejemplos del texto de Heródoto que confirman el empleo de formas verbales expresando orden temporal o Taxis (1976:25-27). Tras un análisis de oraciones temporales y participios, viene a concluir que el tema de presente es portador formal del significado de simultaneidad y el tema de aoristo del significado de anterioridad. Incluye un notable intento de explicación y sistematización de los empleos excepcionales. Tesis semejantes a las de HETTRICH pueden hallarse en el estudio de RIJKSBARON sobre las subordinadas temporales y causales. He elaborado la siguiente descripción siguiendo a estos autores, así como algunas observaciones aisladas halladas en los manuales al uso.

${ }^{6}$ Cf. STAHL 1907:164-5.

7 Dejo de lado los casos de cita directa y algunos empleos de formas temporales con traslación del centro deíctico; cf. KÜHNER-GERTH 1904:360; SMYTH 587.
} 


\section{INDICATIVO 8 :}

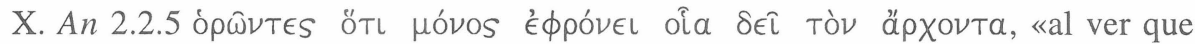
sólo él poseía la sensatez que debe tener un jefe».

Od 17.120 єlı

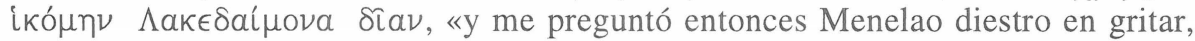
qué necesidad me había hecho venir a la divina Lacedemonia» .

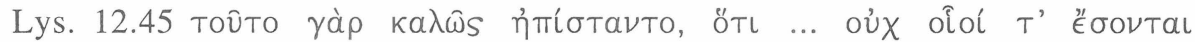
$\pi \epsilon \rho \iota \epsilon \mathcal{\epsilon} \sigma \theta \alpha$, «Pues sabían bien que no serían capaces de prevalecer».

\section{OPTATIVO9:}

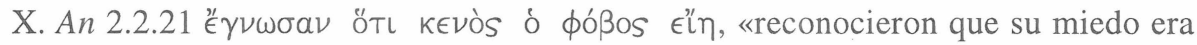
vano».

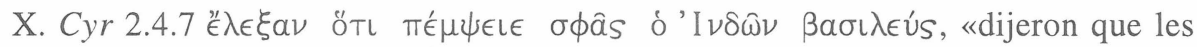
había enviado el rey de los indios».

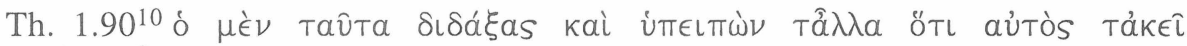

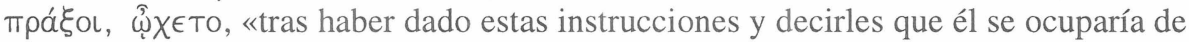
lo demás allí, partió».

1.1.2. ORACIONES DE RELATIVO ${ }^{11}$. En las oraciones adjetivas de relativo aparecen formas de aoristo de anterioridad, presente de simultaneidad y futuro de posterioridad.

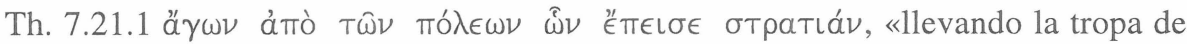
las ciudades que había convencido».

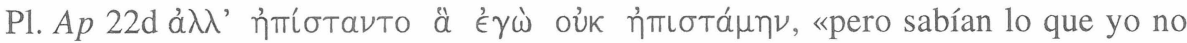
sabía».

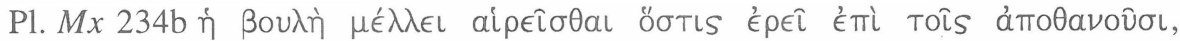
«el consejo va a nombrar a quien ha de pronunciar un discurso sobre los muertos».

\subsubsection{ORACIONES ADVERBIALES.}

1.1.3.1. ORACIONES TEMPORALES Y CAUSALES. En las oraciones temporales y

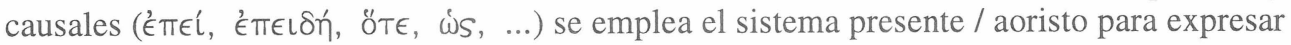

8 KÜHNER-GERTH 1898:359-60.

9 KÜHNER-GERTH 1898:361.

10 HUMBERT 1960:188.

11 RIJKSBARON 1984:88 ss. no hace referencia a a la presencia de tiempo relativo en estas oraciones. Sí STAHL. Con el verbo en futuro, la oración de relativo tiende a asumir una función semántica de FINALIDAD. 
el orden temporal: el presente expresa simultaneidad y el aoristo expresa anterioridad del hecho en relación con la predicación principal ${ }^{12}$. El mismo sistema aparece en las causales con ŐTL, SเóTL 13 .

\section{INDICATIVO ${ }^{14}$ :}

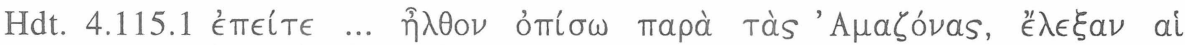

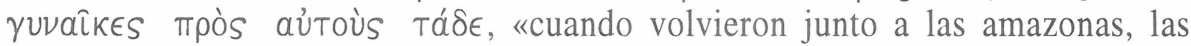
mujeres les dijeron lo siguiente».

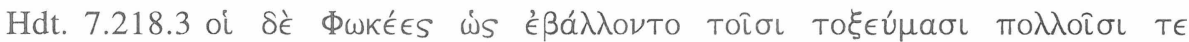

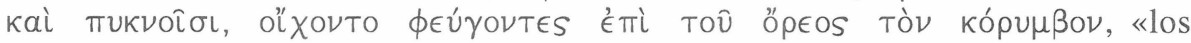
focenses, cuando estaban siendo bombardeados con dardos numerosos y constantes, huían hacia la cima del monte».

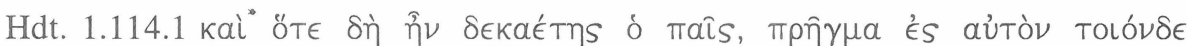

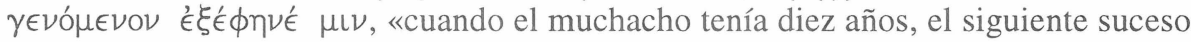
que le aconteció reveló su carácter».

\section{SUBJUNTIVO 15 :}

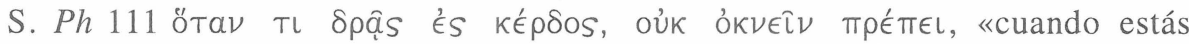
haciendo algo con vistas a un beneficio, no es conveniente vacilar».

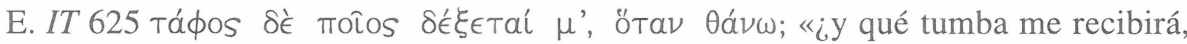
cuando haya muerto?».

\section{OPTATIVO ${ }^{16}$ :}

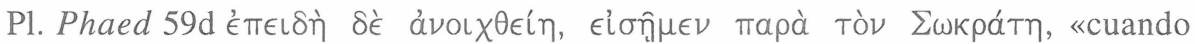
(cada vez que) se había abierto la puerta, entrábamos junto a Sócrates».

\footnotetext{
12 RIJKSBARON 1984:76. Cuando la oración temporal refiere un hecho posterior al hecho referido por la oración principal, se emplean las conjunciones $\tilde{E} \omega$ s «hasta que» y $\pi \rho \mid \nu$ «antes de que» (RIJKSBARON 1984:77). 13 RIJKSBARON 1984:86; cf. KÜHNER-GERTH 1898:169. Cuando la causal refiere un hecho posterior al hecho referido por la oración principal, se tiñe de subjetividad. Entonces se alega como causa el hecho de que se prevé, se sospecha, se teme, se espera que algo acontezca. Puede expresarse mediante conjunción, una

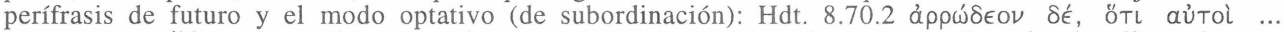

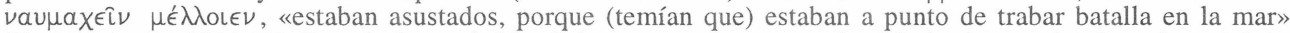
(RIJKSBARON 1984:87). Pero la forma habitual de expresar estas causales "de hecho previsto" es el participio

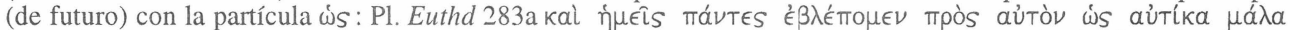

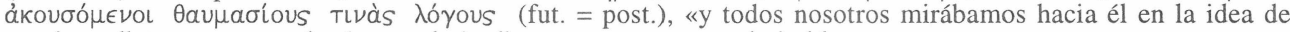
que inmediatamente escucharíamos algún discurso sumamente admirable»

14 RIJKSBARON 1984:76 SS.. Cf. HETTRICH 1976.

15 STAHL 1907:167 ss.; RIJKSBARON 1984:84.

16 STAHL 1907:174; RIJKSBARON 1984:85.
} 


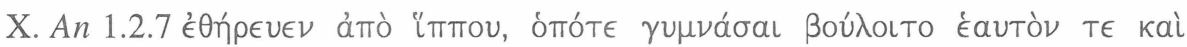
Toùs "ாтाоus, «cazaba a caballo, cada vez que deseaba ejercitarse a sí mismo y a sus caballos».

1.1.3.2. ORACIONES CONDICIONALES. En las oraciones condicionales y concesivas no parece expresarse sistemáticamente el orden temporal entre prótasis y apódosis. RUIJGH (1971) sugiere que sí se expresa el orden temporal y ofrece una exposición detallada del sistema (ao/pres) con ayuda de ejemplos fingidos. Pero es difícil encontrar en las gramáticas testimonios y ejemplos que apoyen su tesis. En cambio, son abundantes los contraejemplos ${ }^{17}$.

1.1.4. INFINITIVOS. El orden temporal se expresa sólo en los infinitivos declarativos ${ }^{18}$ o de "estilo indirecto"19, dependientes de verbos de lengua y pensamiento. El sistema incluye aoristo con valor de anterioridad, presente con valor de simultaneidad y futuro con valor de posterioridad ${ }^{20}$.

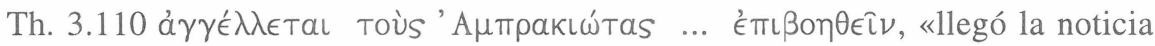
de que los ampraciotas acudían a ayudar».

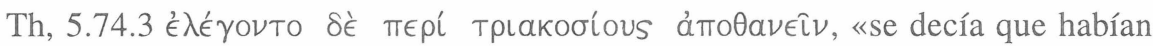
muerto alrededor de trescientos».

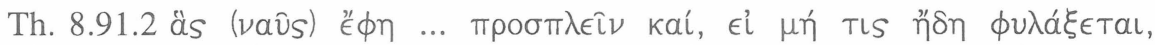

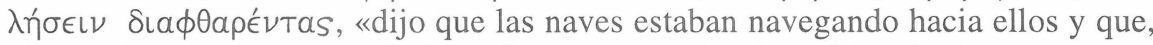
si no se instalaba una guardia inmediatamente, serían destruidos sin darse cuenta».

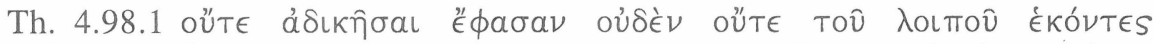
$\beta \lambda a ́ \psi \epsilon \iota \nu$, «afirmaron que ni habían profanado (el santuario) en nada ni en adelante le causarían daño voluntariamente».

1.1.5. PARTICIPIOS. El participio puede expresar orden temporal en todos sus empleos sintácticos, atributivo y predicativo ${ }^{21}$.

Th. 3.58.5 Ëं creyendo que los colocaba en tierra amiga».

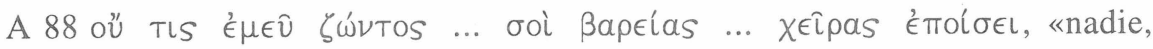
mientras yo viva, te pondrá las manos encima».

17 Cf. STAHL 1907:173; cf. etiam RIJKSBARON 1984:68 ss.

18 Cf. STORK 1984; MARTÍNEZ VÁZQUEZ 1989.

19 SMYTH 1963:449.

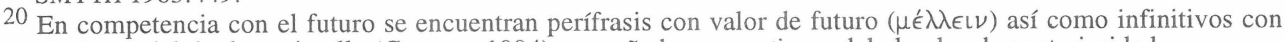
partícula modal, incluso sin ella (CRESPO 1984), que añaden un matiz modal al valor de posterioridad.

21 STAHL 1907:209, 211, 218; RIJKSBARON 1984:104. 


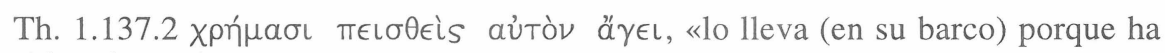
sido sobornado».

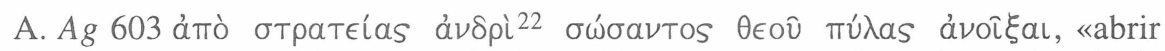
las puertas al marido, tras haberlo traído a salvo un dios, de vuelta de la expedición».

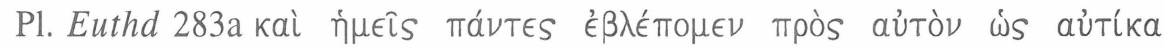

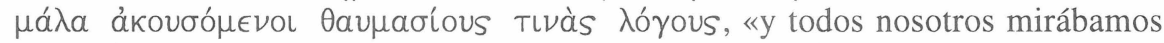
hacia él en la idea de que inmediatamente escucharíamos algún discurso sumamente admirable».

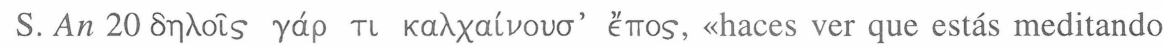
una respuesta».

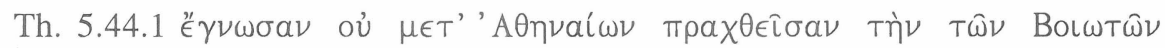

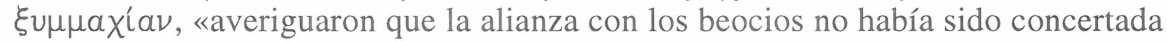
con los atenienses».

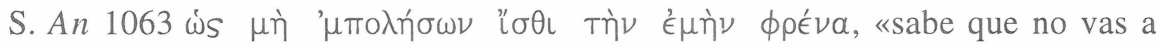
traficar con mi voluntad».

1.2. ULTERIOR DELIMITACIÓN DEL ÁMBITO. En la descripción anterior se aprecia cómo el orden temporal se expresa en determinados tipos de oraciones subordinadas. Completo ahora la descripción con una relación de casos en que el orden temporal no se expresa. Se trata bien de situaciones excepcionales bien de restricciones de índole semántica que afectan a algunos de los tipos de subordinación descritos más arriba.

1.2.1. SITUACIONES EXCEPCIONALES ${ }^{23}$. Hay que precisar, en primer lugar, que el orden temporal es una posibilidad de determinación de una predicación con referencia a otra predicación. El autor puede renunciar a esta posibilidad e introducir en la predicación subordinada un operador de tiempo deíctico, matizado frecuentemente por medio de un satélite temporal:

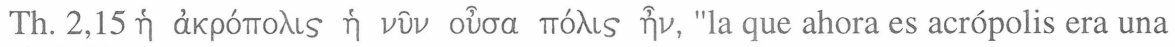
ciudad".

Ya he avanzado la idea de que el orden temporal es noción propia de la predicación subordinada. Parece coherente la idea de que, para que la relación temporal entre dos predicaciones se gramaticalice y se exprese sistemáticamente, es necesario que exista una relación gramatical, sintáctica entre estas predicaciones. Si esta hipótesis es correcta, vano será buscar nociones de orden temporal en la predicación independiente, donde sólo

$22 \not ̋ \nu \delta \rho a$ (PAGE).

23 Véase HETTRICH 1978:38-39; OGUSE 1962:59. A propósito de empleos aparentemente excepcionales (incidencia de la negación, iteración y de la semántica verbal), véase HETTRICH 1978:45-83. 
aparecerán como implicación contextual, que emana conjuntamente de rasgos gramaticales y datos contextuales. Esta hipótesis dará razón del hecho de que la expresión del orden temporal "falle" en los casos de subordinación inversa ${ }^{24} \mathrm{o}$ "falsa" subordinación.

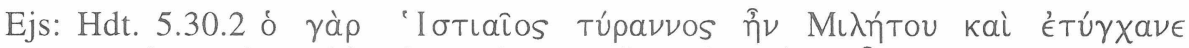

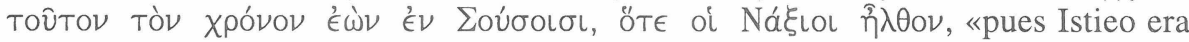
tirano de Mileto y coincidió que se encontraba en Susa cuando llegaron los naxios»;

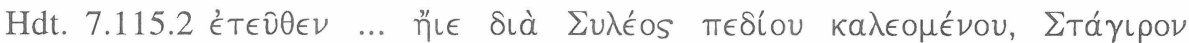

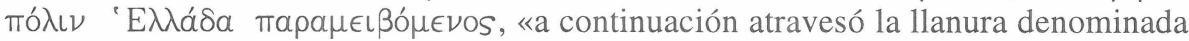
Sileo y pasó por junto a la ciudad griega de Estagiro».

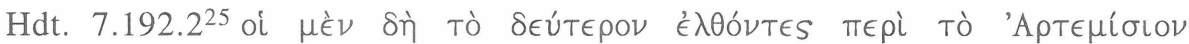

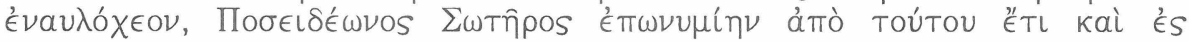

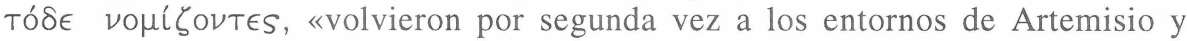
acamparon allí, dando el epónimo de salvador a Poseidón desde entonces y aún hasta la actualidad».

1.2.2. RESTRICCIONES SEMÁNTICAS. Es éste un factor muy influyente en la expresión del orden temporal y sorprendentemente no he hallado apenas referencias sobre él. Es claro que las nociones de orden temporal sólo son complementarias de determinadas funciones semánticas de la predicación y que son incompatibles con otras. Además de la función semántica propiamente dicha tiene una relevancia la relación semántica concreta entre el predicado regente y el regido. Es de sentido común establecer las siguientes observaciones generales. Porque el orden temporal se expresa sólo allí donde se puede expresar y para que ello sea posible se requiere: 1) que los dos hechos referidos sean distintos. 2) Que la relación temporal entre los dos hechos no esté determinada por otros factores.

1.2.2.1. Es necesario que las dos predicaciones en relación sintáctica refieran hechos distintos y no refieran conjuntamente un mismo hecho. De otro modo carece de sentido expresar la relación temporal entre los hechos. Dicho en otros términos, la correferencialidad de predicación principal y predicación subordinada no es compatible con la expresión del orden temporal. Esta correferencialidad es bastante frecuente ${ }^{26}$. HETTRICH (1976:77 ss.) habla de tres subtipos, que yo reduciría a dos.

Un tipo de construcción libre, que se da tanto con participios como con formas personales ${ }^{27}$ :

24 HETTRICH (1976:32 ss.) habla de "participios inversos". Compárese, en todo caso, STAHL 1907, ya citado, que deja al margen las parathetische Nebensätze. Respecto a los participios, cf. asimismo STAHL 1907:216.3.

25 HETTRICH 1976:39.

26 Cf. RUIJGH 1971:251. Y mucho antes, STAHL 1907:169.1, 171.1 y para el participio, 1907:212-3, 220.1.

27 Los verbos de percepción construidos con participio predicativo son un caso interesante. Habría que

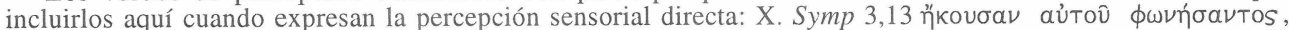
«le oyeron hablar», donde el aoristo no expresa anterioridad. Pero se integran en el uso normal cuando expresan percepción indirecta (Cf. OGUSSE 1962:48-49). 


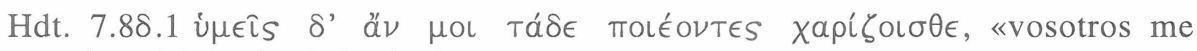
complaceríais haciendo lo siguiente».

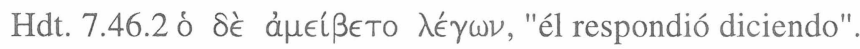

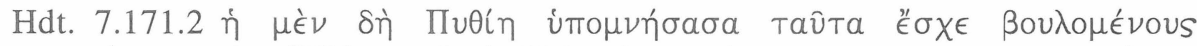

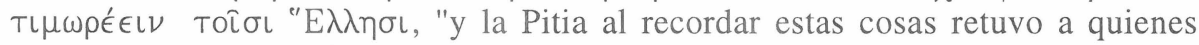
querían vengar a los griegos".

Es mucho menos frecuente la or. subordinada correferencial con verbo personal:

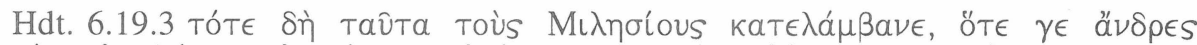

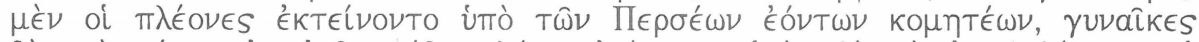

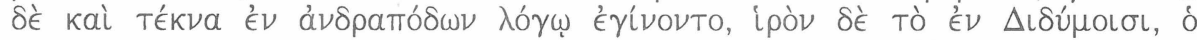

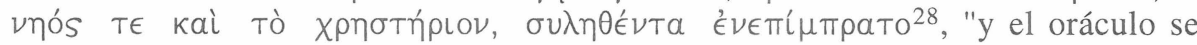
cumplió en los Milesios entonces, al ser muertos la mayoría de los hombres por los persas de larga cabellera, ser esclavizados las mujeres y los niños y ser incendiados tras ser saqueados el centro sagrado de Didima, el templo y la sede oracular".

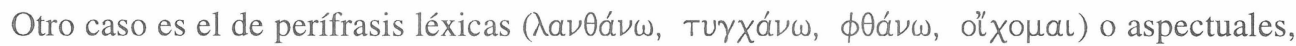
con participio:

Hdt. 7.218.3 o"x

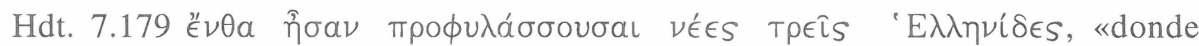
estaban montando guardia como avanzadilla tres naves griegas».

Se observa en estos casos una tendencia de las dos formas verbales a expresar conjuntamente el aspecto, ocurriendo en el mismo tema aspectual.

1.2.2.2. La relación temporal entre dos hechos referidos por predicaciones ligadas sintácticamente puede estar determinada por factores que anulan toda posibilidad de que el verbo exprese orden temporal.

\subsection{Un factor determinante puede ser el valor semántico concreto del verbo regente.}

Es el caso de los verbos de la percepción directa. No se puede tener percepción directa de un hecho que ya ha acontecido o aún ha de acontecer. Consecuentemente la lengua no se esforzará en expresar la relación temporal entre el acto de percepción y el hecho percibido.

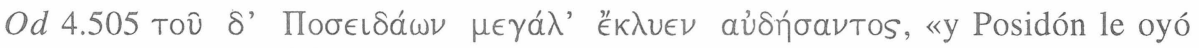
emitir sus fuertes palabras».

\footnotetext{
${ }^{28}$ Ejemplo tomado de HETTRICH 1978:79, quien lamenta que SCHWYZER, CHANTRAINE y HUMBERT se sirvan de ejemplos como éstos para negar la existencia de tiempo relativo en griego. Cf. infra.
} 


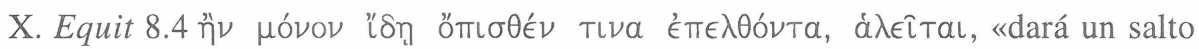
tan sólo con que vea que uno se le acerca por detrás» ${ }^{29}$.

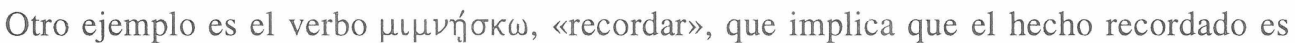
anterior al acto del recuerdo, de modo que el hecho recordado puede expresarse en no importa qué tema aspectual, sin expresar el orden temporal, ya fijado por el verbo:

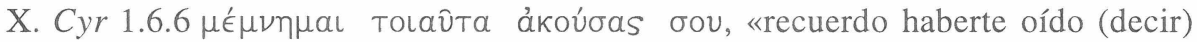
tales cosas»

pero

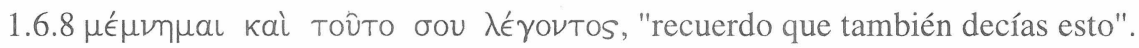

Con todo hay que decir que es la construcción normal de este verbo: participio aoristo ligado al sujeto ("oir", etc.) y participio presente ligado al objeto ("decir", etc.):

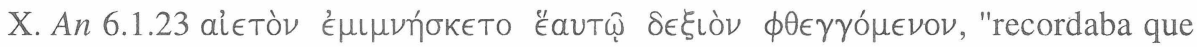
un águila había gritado a su derecha" (no tiene sentido «...que estaba gritando a su derecha»).

1.2.2.2.2. En el comienzo de esta sección he hecho referencia a la relevancia de la función semántica de la predicación subordinada. En efecto, determinadas funciones semánticas implican un orden fijo o un cierto grado de correferencialidad con la predicación principal y, consecuentemente, anulan la expresión del orden temporal. En las oraciones finales y consecutivas no se expresa el orden temporal porque éste es secuencial: se entiende que, en todo caso, el hecho referido por la subordinada, a saber, el fin o la consecuencia, es posterior al hecho referido por la principal, pero no coexiste con él ni lo precede. La presencia de un futuro en algunas finales sería, todo lo más, redundante.

Este fenómeno es especialmente significativo en los participios circunstanciales, que pueden ejercer funciones distintas y, por tanto, unas veces expresan el orden temporal y otras veces no lo hacen. Así, los participios temporales y causales, a veces también los condicionales y concesivos, expresan el orden temporal regularmente (cf. supra). Por ello los genitivos y acusativos absolutos, que suelen ejercer una de estas funciones, típicamente expresan el orden temporal. Pero, como es bien sabido, el participio puede expresar también la función de medio o MANERA. Pues bien, cuando el participio refiere un hecho que constituye la forma en que se desarrolla el hecho referido por la predicación principal, en realidad no hace referencia a un hecho independiente de aquél, sino que hace referencia a ese mismo hecho, en todo caso a una fase de éste. Dicho en otros términos, la función de MANERA implica un grado de correferencialidad de participio y predicación principal. Obsérvese el ejemplo siguiente, donde el participio, con función de MANERA, viene a ser una extensión, una explicación del verbo principal: 


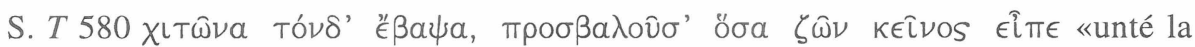
túnica aplicándole cuantas cosas me había dicho ...» ${ }^{30}$.

Es por ello que aquí el participio de aoristo no expresa un hecho anterior al hecho expresado por el verbo principal: se trata del mismo hecho. Merece la pena reseñar que este participio modal es típicamente un participio concertado con el sujeto del verbo principal. Evidentemente, si son correferenciales, han de tener el mismo sujeto.

1.2.2.3. Una última posibilidad, más excepcional, es el caso en que el operador de tiempo relativo es introducido por un satélite temporal. Constituye una codificación alternativa del orden temporal a la codificación mediante el mero tema verbal. Éste queda entonces relagado a un segundo plano, expresando a lo sumo una matización aspectual:

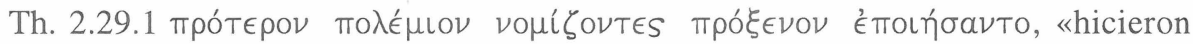
embajador a quien antes tenían por enemigo».

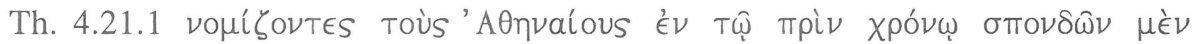
É $\pi \imath \theta u \mu \epsilon \hat{\imath} \nu$, «convencidos de que los atenienses deseaban ya antes una tregua».

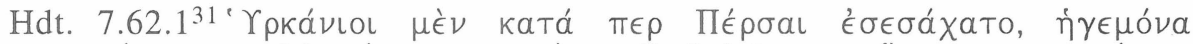

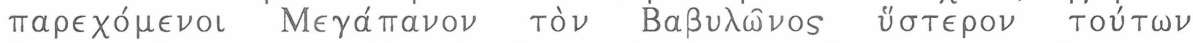

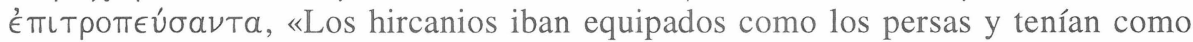
jefe a Megápano, que posteriormente fue administrador de Babilonia».

\section{INTERPRETACIONES DE LOS HECHOS DESCRITOS.}

2.1. Algunos autores niegan abiertamente que el verbo griego exprese en absoluto el orden temporal. KÜHNER-GERTH, WACKERNAGEL y SMYTH sostienen que los temas verbales griegos pueden expresar indistintamente acciones anteriores, simultáneas o posteriores a la acción referida por el verbo principal ${ }^{32}$, que el griego, por ejemplo, no tiene una forma especial para expresar tiempo que es anterior al pasado ${ }^{33}$. HOLT, por su parte, es tajante al respecto: Lo que con frecuencia se ha afirmado concerniente a un pasado relativo designado por el infinitivo y el participio de aoristo es enteramente falso (1943:18). La clave de su particular visión está en el hecho de que no distinguen las circunstancias en que estas nociones se expresan sistemáticamente de las circunstancias en que no es así. Veamos con algún detalle dos exponentes claros de esta tesis.

2.1.1. Defiende la tesis HUMBERT en su manual de sintaxis griega. En su opinión, el que creamos ver en los temas verbales griegos un expresión del tiempo relativo se debe a la analogía con el latín o nuestras propias lenguas:

\footnotetext{
30 MOOREHOUSE 1982:212.

31 HETTRICH 1976:38.

32 KÜHNER-GERTH, 1904:145, 151, 169, 188. WACKERNAGEL 1928:151 ss. SMYTH 1963:\$1866-7, §1874, $\S 1888, \S 1907, \S 1944$.

33 SMYTH 1963:§1907.
} 
Mais il y a plus: le grec n'exprime pas le temps relatif —c'est-à-dire ni l'anteriorité relative dans le passé ou l'avenir, ni la simultaneité. Autrement dit, les temps du grec ont gardé, les uns par rapport aux autres, cette autonomie caractéristique de l'état de choses le plus ancien: nous en sommes très souvent gênés parce que l'analogie du latin ou de notre langue nous entraîne à voir une notation du «temps relatif», alors que le temps en question se justifie par lui-même. (1960:133)

Los empleos de los temas verbales se explican, según HUMBERT, por su valor aspectual. Ciertamente el valor aspectual ha de estar en la base de estos empleos. Pero él se muestra excesivamente tajante cuando pretende justificar la ausencia de orden temporal en griego por el temperamento intuitivo del pueblo griego:

Langue d'un peuple à tempérament intuitif, le grec ne s'est jamais soucié d'exprimer ces rapports abstraits: il cherche au contraire à se présenter les modalités de l'action dans son devenir et par rapport à celui qui agit. Le grec est sensible à l'aspect, parce que celui-ci est concret et subjectif. (1960:133-4)

Desde esta perspectiva se empeña en explicar todos los ejemplos en relación con el valor aspectual del tema. Básicamente describe los mismos usos que he descrito más arriba. Emplea como contraejemplos los casos excepcionales o afectados de restricción semántica, que reciben tratamiento de empleos normales, y con ellos arguye que el valor aspectual es el único relevante.

Así, en el caso del participio, reconoce que existen concordancias entre la simultaneidad y el participio de presente y entre la anterioridad y el participio de aoristo (1960:170). Pero insiste en que se trata de un valor aspectual: si el participio de presente expresa frecuentemente nuestra simultaneidad -viene a decir-es porque la acción que expresa está contemplada en su desarrollo (1960:170):

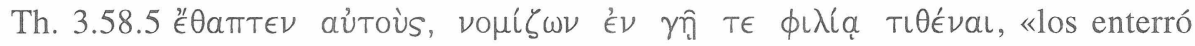
creyendo que los colocaba en tierra amiga».

Otras veces el participio de presente expresa la anterioridad real:

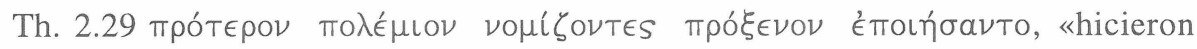
próxenos a quien antes consideraban enemigo».

HUMBERT presenta en el mismo plano empleos de participios que se hallan en circunstancias muy diferentes. Cierto es que el participio de presente señala aquí un hecho anterior al hecho señalado por la predicación principal. Pero la idea de anterioridad es una implicatura contextual, pues sin el adverbio temporal $\pi \rho \delta ́ т \epsilon \rho o \nu$, la tendencia general sería a interpretar el valor del participio como de simultaneidad (cf. supra):

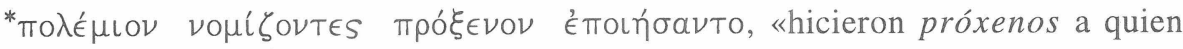
consideraban enemigo». 
En el siguiente pasaje muestra HUMBERT cómo en una misma frase aparecen participios de aoristo y de presente igualmente simultáneos respecto al verbo principal:

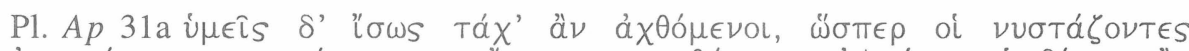

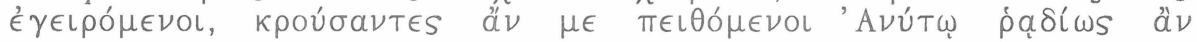

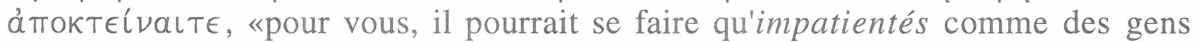
somnolents qu'on réveille, vous fiant à Anytos dans un mouvement de colère, vous

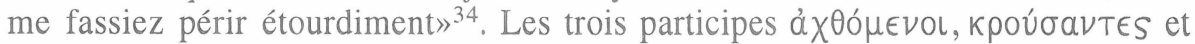
$\pi \epsilon \iota \theta$ ó $\mu \epsilon \nu$ ol sont, pour nous, également simultanés par rapport à l'aoriste

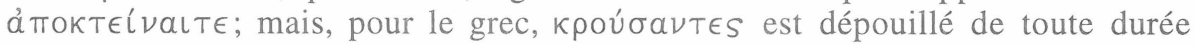
-nuance finement rendue dans la trad. de Croiset. (1960:171)

En mi opinión no son igualmente simultáneos. HUMBERT coloca en un mismo plano participios que constituyen tipos semánticamente distintos. Como fielmente refleja la

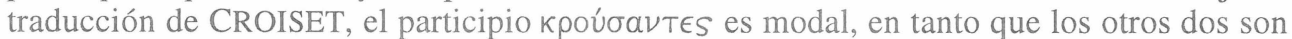
causales y, en consecuencia, es parcialmente correferencial con el verbo regente. Recuérdese que el texto continúa en la línea de la imagen del tábano y el caballo introducida poco antes.

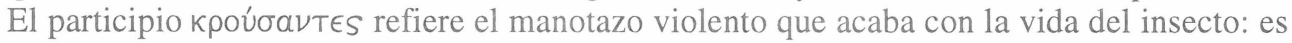
parte de esa acción.

El participio de aoristo estaría igualmente determinado por su valor exclusivamente aspectual, de ahí que pueda expresar tanto la simultaneidad como la anterioridad (1960:172). Cita una serie de ejemplos de aoristos de simultaneidad, todos los cuales son correferenciales con sus verbos regentes (1960:173). Además de las perífrasis con $\lambda a \nu \theta a ́ v \omega, \phi \theta a ́ \nu \omega$,

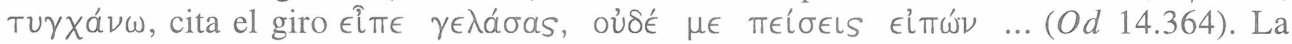
cuestión es si estas expresiones reflejan el uso general o constituyen un caso aparte, especial. Yo me inclino por la segunda opción.

Con todo, y a pesar de lo reseñado hasta ahora, HUMBERT reconoce que el participio expresa tiempo cuando expresa acciones que salen de la zona de influencia del verbo principal (1960:171), esto es, acciones independientes del verbo principal (1960:175). Se refiere a los participios que corresponden a proposiciones de relativo, temporales, causales o concesivas. El empleo recibe tratamiento de uso especial y como única explicación se menciona que el participio equivale a un indicativo de presente o aoristo, según el caso. Parece un reconocimiento implícito de los valores que he descrito más arriba, pero todo queda en el aire: no hay referencia explícita a si se trata de tiempo absoluto o relativo y tampoco se explica el valor de los temas aspectuales en estos ejemplos. Algún ejemplo, incluso, no coincide con la explicación que da, porque no corresponde a indicativo alguno. Así en:

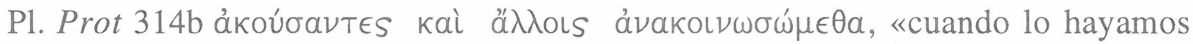
escuchado, haremos a otros partícipes».

34 «Pero quizá vosotros, fastidiados acaso como los que están sesteando y los despiertan, vayáis de un manotazo, persuadidos por Anito, a matarme fácilmente» trad. de GARCIA CALVO. 


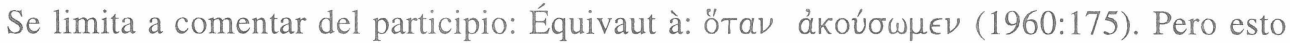
no es un indicativo. No se entiende qué valor temporal tendría, ni cómo se explicaría ese valor temporal, según HUMBERT. Es difícil percibir otro valor que el de orden temporal:

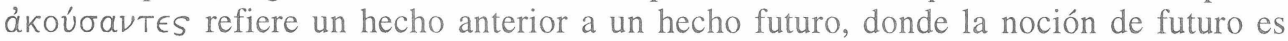
expresada por el verbo regente y la noción de anterioridad es expresada por el tema aspectual del participio, que aquí no expresa simplemente aspecto.

Sin embargo en su descripción de los valores puramente temporales del participio de futuro -sin valor de intención u obligación-, vuelve a recurrir a la tesis de que el participio expresa tiempo en virtud de su equivalencia con una forma de indicativo y, ahora sí, afirma expresamente que se trata de tiempo absoluto:

Mais ces valeurs quasi-modales et subjectives étaient irrésistiblement entraînées vers le temps, c'est-à-dire vers le futur. Celui-ci ne peut exprimer que le temps absolu, puisqu'il ne comporte pas ces aspects qui ont au moins l'air, dans les thèmes de présent et d'aoriste, d'avoir quelque rapport avec notre temps relatif. C'est ainsi que l'on passe, de tournures qui ont una apparence désidérative, mais sont déjà entrées dans le temps, à des futurs uniquement temporels, équivalant à des futurs de l'indicatif. (1960:176)

Un ejemplo:

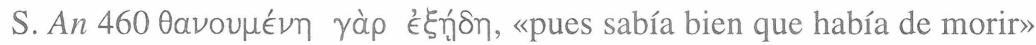

donde el participio expresa un hecho posterior a otro hecho pasado. Para justificar el valor de tiempo futuro (absoluto) que le atribuye, HUMBERT añade: c'est-à-dire, «je savais bien ceci: je mourrai» (ibidem).

2.1.2. ADRADOS (1992) niega así mismo con rotundidad que el tiempo relativo constituya una categoría gramatical en griego:

Así, el tiempo relativo es simplemente un derivado del aspecto y un hecho de frecuencia que debe ser apoyado por el contexto; cuando éste apunta en sentido contrario, la regla habitual puede invertirse. (1992:439)

Más adelante, concluye:

Así, en definitiva, el aspecto no tiene directamente nada que ver con el tiempo relativo o «taxis»y es perfectivamente (sic) distinguible del tiempo presente, pretérito y futuro de los indicativos. Pero la combinación de aspecto y contexto facilita la ordenación de las acciones o procesos de los verbos de un pasaje con relación unas a otras. El aspecto da a priori posibilidades de frecuencia, pero éstas son modificadas en ocasiones por el contexto. Así, los conceptos de simultaneidad, anterioridad, inceptivo, árctico (designando el comienzo de la acción) y demás deben separarse cuidadosamente de la definición del aspecto y aun de la del tiempo 
gramatical del verbo griego. Es interesante, sin embargo, comprobar cómo tiempo, aspecto y contexto juegan entre sí para indicar toda clase de matices en la organización de la oración compleja. (1992:441-2)

Las palabras de ADRADOS son reflejo de cómo, a veces, la interpretación de los hechos lingüísticos depende estrechamente de la actitud que a priori se adopta respecto a ellos. En realidad ADRADOS reconoce la existencia de estos valores:

Efectivamente, en exposiciones tradicionales era habitual la doctrina de que el part. pres. indica simultaneidad, el de aor. anterioridad; (...) La regla general, a la que ya aludimos más atrás, es cierta y no necesita mucha ejemplificación. (1992:441)

Pero entiende que estas nociones deben separarse de los valores gramaticales de tiempo y aspecto porque son sólo hechos de frecuencia necesitados de apoyo contextual. ¿Y qué son el tiempo y el aspecto, y, en definitiva, toda categoría gramatical, sino hechos de frecuencia necesitados de apoyo contextual? ¿No es cierto que una categoría gramatical realiza el valor que le es propio por sistema, siempre y cuando el contexto no lo neutralize? Así, por ejemplo, el indicativo de aoristo tiene valor de tiempo pasado, pero no en máximas y sentencias. El presente actual, único presente en sentido estricto, tiene una frecuencia relativa muy baja, comparado con otros usos: presente general, presente histórico.

La diferencia de percepción estriba en la relevancia que se otorgue a los contraejemplos. Si se consideran usos normales, debe negarse la categoría. Si se consideran posiciones de neutralización, que pueden explicarse y describirse conjuntamente con los usos "normales", entonces no hay motivo para negar el valor gramatical. Los ejemplos que aduce ADRADOS encajan en los tipos descritos más arriba como excepcionales.

Entre los ejemplos de casos especiales (sic) incluye tres de participio presente referido a una acción anterior. Todos ellos acompañados de una expresión adverbial a la que se debe el sentido de anterioridad, como en:

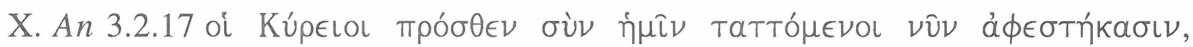
«Los cirios, que antes formaban con nosotros, ahora han desertado» (1992:441).

En otros casos no advierte que el participio es correferente del verbo regente, lo que imposibilita que el aoristo exprese anterioridad:

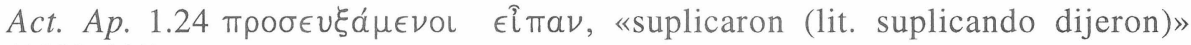
(1992:441).

\subsection{TEORIAS QUE EXPLICAN EL TIEMPO RELATIVO}

2.2.1. TEORIA DE LA TRANSFORMACION. El hecho de que los temas verbales expresen orden temporal en las construcciones conocidas como de estilo indirecto en sentido amplio es explicado por varios autores a partir de transformaciones de estilo directo a estilo 
indirecto, esto es, en la idea de que el estilo indirecto reproduce los tiempos del estilo directo. Así, por ejemplo, STAHL afirma de los optativos oblicuos del discurso indirecto que reproducen la relación temporal del discurso directo, ya pueda estar éste en lugar del indicativo o en lugar del subjuntivo. En presente representa éste el tema de presente del discurso directo, i.e., el indicativo del presente y del imperfecto o el subjuntivo de presente en sus diversas relaciones temporales, en perfecto, corresponde al tema de perfecto, en aoristo se halla en lugar del indicativo o subjuntivo de aoristo, en futuro, por el indicativo de futuro (1907:176).

Ofrece la misma explicación a propósito de infinitivos dependientes de verba dicendi y putandi: el infinitivo dependiente, regido por verba dicendi o putandi, reproduce de nuevo la relación temporal del discurso directo exactamente como el optativo oblicuo (...) El infinitivo de presente y perfecto designa entonces simultaneidad (presente relativo) o anterioridad duradera (dauernde Priorität), el infinitivo de Aoristo, anterioridad (pasado relativo) en sí. El futuro relativo puede ser expresado, además del futuro, mediante el

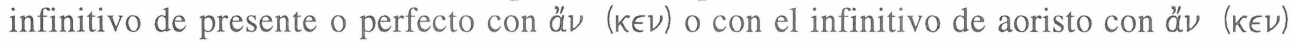
(1907:188 ss.). Idénticas palabras encontramos de nuevo a propósito del participio predicativo dependiente de verba sentiendi, a veces, de verba affectuum (1907:218 ss.).

En la misma línea que STAHL se expresa DELBRÜCK (Vergl. Synt. II, 274), al sostener que los infinitivos dependientes de verbos de lengua y pensamiento han devenido infinitivos a partir de formas aumentadas al cambiarse la forma de discurso (bei Umwandlung der Redeformen aus Augmentformen zu Infinitiven geworden sind). Y BRUGMANN (Gr. Gr ${ }^{3}$ 521) que entiende que estos infinitivos, así como los optativos oblicuos, son reflejos de los indicativos del discurso directo. HENTZE ${ }^{35}$ se permite señalar las construcciones típicas en que pudo tener su origen esta "transferencia" de sentido temporal del indicativo al infinitivo ${ }^{36}$.

Puede admitirse esta explicación en determinadas construcciones de verbos de lengua, en que el locutor pretende reflejar indirectamente un discurso emitido por una persona concreta en una ocasión concreta. Dado que en dependencia de un verbo de lengua se admite tanto la cita directa como distintas formas de cita indirecta, inevitablemente se dan equivalencias:

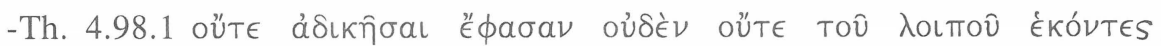
$\beta \lambda \alpha ́ \psi \in \iota \nu$.

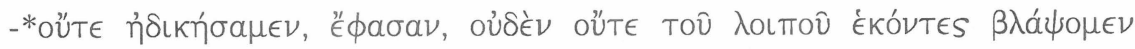

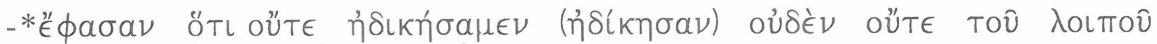

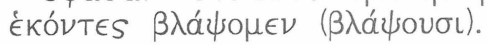

Pero cuesta creer que, cada vez que un griego empleara el infinitivo de aoristo tras un verbo de lengua, necesariamente pensara en reflejar un indicativo de aoristo del discurso directo, y 
no se limitara a indicar con ello que lo dicho tuvo lugar con anterioridad al momento de su enunciación. No he hallado ejemplos probatorios en este sentido, pero el pasaje siguiente puede dar una idea de lo que pretendo dar a entender:

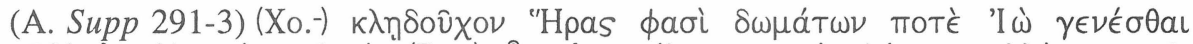

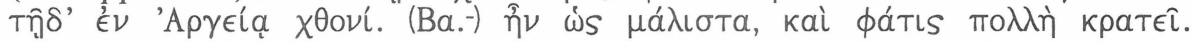
«CORIFEO- Dicen que en otro tiempo Io fue guardiana del templo de Hera en esta tierra argiva. REY- Lo era, muy cierto, fama extendida impera».

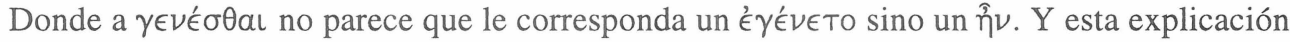
resulta menos verosímil a medida que se extiende a verbos de pensamiento, percepción y sentimiento. Se me antoja un tanto gratuito e innecesario acudir a la imagen de padre y

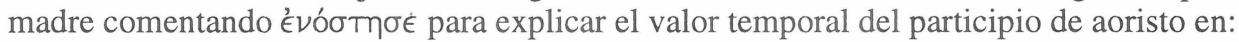

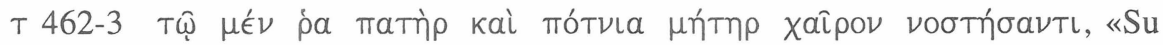
padre y su augusta madre se alegraron de que hubiera vuelto».

Por otra parte hay que decir que ningún autor, al menos que yo sepa, ha pretendido aplicar el mecanismo de la transformación de estilo directo en estilo indirecto a la explicación de la expresión del orden temporal como fenómeno en su conjunto. El propio STAHL (1907:105), fiel exponente de esta tesis tan presente en los manuales, explica las nociones de orden temporal en oraciones adverbiales (temporales, causales), oraciones de relativo y participios circunstanciales a partir del valor aspectual de los temas verbales, sin acudir a transformaciones de tipo alguno (Cf. infra).

2.2.2. TEORÍAS DE LA TEMPORALIDAD. Agrupo bajo este epígrafe todas aquellas teorías que explican los valores que nos ocupan como realizaciones de una sola categoría, que suele definirse como temporalidad y engloba las nociones que hemos agrupado en tres categorías diferentes, de aspecto, tiempo y orden temporal. Evidentemente, el punto de partida de esta categoría suele ser una definición muy abstracta, incluso abiertamente ambigua, en la que, como rasgo formal, yo destacaría la presencia abusiva de disyunciones, es decir, de fórmulas del tipo $\mathrm{X}=\mathrm{Y}$ o bien $\mathrm{Z} \ldots$ o bien $\mathrm{N}$.

Ejemplo de este tipo de teoría puede apreciarse en la definición que KURYLOWICZ ofrece de la categoría de orden temporal (anteriority), cuya relación con el tiempo verbal (tense) es oscura:

the reference of an action, wether present or past, etc., to a certain moment: the moment of speaking or a past one, etc. (1964:90)

Casi a renglón seguido sostiene que anterioridad puede ser una variante contextual independiente del aspecto perfectivo o imperfectivo de la forma verbal, hecho que atribuye al griego, negando que el orden temporal (anteriority) sea relevante en esta lengua. Y, sin embargo, más adelante en su estudio, se refiere al aoristo griego como ejemplo de forma que 
expresa perfectividad como función primaria y anterioridad como función secundaria, y escribe:

Greek $\mathscr{E}^{\prime} \gamma \rho a \psi \alpha$ is in the first place perfective as against É $\rho \alpha \phi o \nu$, but expresses anteriority with reference to the present $\gamma \rho \alpha \dot{\phi} \omega .(1964: 97)$

Donde es evidente que ahora la anterioridad se toma en el sentido en que hemos definido el tiempo deíctico y no en el de orden temporal, pues la oposición entre $\epsilon^{\prime} \gamma \rho a \psi \alpha$ y $\gamma \rho a ́ \phi \omega$ es una oposición entre tiempo pasado y tiempo presente.

Mas voy a centrarme en el análisis de la teoría que considero más significativa de cuantas se han expuesto específicamente sobre el verbo griego. Se trata de un reciente trabajo de RUIJGH (1991).

En primer lugar describe los valores generales de los cinco temas verbales, que él denomina temas temporales (1991:199): tema de presente, t. de aoristo, t. de futuro, t. de perfecto, t. de futuro de perfecto. En líneas generales define estos valores como relativos a un punto de orientación, que suele denominar moment donné, concepto sumamente ambiguo, pues:

il peut s'agir du moment présent, du moment initial d'une autre action mentionnée dans le contexte ou d'un moment simplement présent dans l'esprit du locuteur. (1991:199)

Nótese que en realidad se trata de tres posibilidades correspondientes a las tres categorías que postulé en mi hipótesis de trabajo y son tesis central de este capítulo. En el primer caso, si el punto de orientación es el momento presente, el valor será temporal, de tiempo deíctico. En el segundo caso, si el punto de orientación es el momento en que tiene lugar otro hecho referido en el contexto, el valor será de orden temporal (si bien yo he añadido la necesidad de que haya una dependencia sintáctica respecto al verbo que constituye el punto de orientación para que la relación se gramaticalice). En tercer lugar, en fin, si el punto de orientación coincide simplemente con un momento presente en el espíritu del locutor, el valor será aspectual.

Los valores fundamentales de los cinco temas temporales serían los siguientes:

-El tema de presente expresa que en un momento dado (por la situación o el contexto), la acción expresada por el tema verbal está todavía en curso, que todavía se halla inacabada (valor imperfectivo).

-El tema de aoristo expresa que el cumplimiento (achèvement) (o el fin) de la acción es anterior a un momento dado (valor confectivo).

-El tema de perfecto expresa que en un momento dado, el estado resultante del cumplimiento de la acción expresada por el tema verbal subsiste aún (valor estáticoperfectivo). 
-El tema de futuro expresa que la acción es posterior al momento presente.

-El tema de futuro de perfecto expresa que el estado resultante del cumplimiento de la acción expresada por el tema verbal es posterior al momento presente.

Añade una representación gráfica:

Pres.

Aor.

Perf.

Fut.

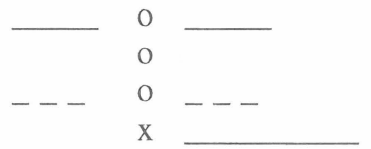

Encuentro en su teoría los siguientes puntos débiles: 1) La definición del futuro como tiempo absoluto rompe la uniformidad de la categoría en los términos (ciertamente vagos) en que parece definirse. Por referirlo gráficamente, la (x) que sustituye a (o) en la línea que corresponde al futuro no es coherente con el sistema descrito. 2) No queda nada clara la relación entre los tiempos expresados mediante desinencia primaria / secundaria y los valores de estos temas "temporales". 3) Como ya he adelantado, la simplificación de valores aspectuales y valores de tiempo relativo descansa sobre una base un tanto resbaladiza.

1) La definición del futuro como tiempo absoluto rompe la uniformidad de la categoría y le obliga a hacer matizaciones cuando habla de los temas "temporales" en su conjunto:

Tandis que les thèmes temporels servent en principe à exprimer le temps relatif (abstraction faite des thèmes de futur), les modes du verbe fini expriment le temps absolu, en outre de leur valeur modale. (1991:200)

Falta una explicación de la relación entre el futuro y los modos, que, en definitiva, expresarían las mismas nociones.

Pero la definición del futuro como tiempo absoluto también es matizada, ya que choca con el hecho conocido de que infinitivo y participio (y optativo) de futuro no expresan tiempo futuro: le thème du futur ... exprime en principe le futur absolu (200). Se apoya en el hecho

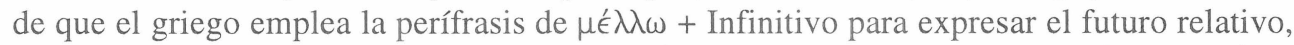
i.e., la posterioridad. Pero termina reconociendo, aunque veladamente, que existen empleos relativos del futuro en infinitivo y participio ${ }^{37}$.

\footnotetext{
37 Lo hace muy veladamente. Del infinitivo de futuro en el estilo indirecto sólo dice que le grec emploie en style indirect le thème temporel qui répond au style direct. Y, a continuación, añade c'est dans ce cadre que s'explique l'emploi final du participe futur (1991:200). Las breves palabras que dedica al particpio en general no aclaran gran cosa: Le plus souvent, il exprime le temps relatif. En principe, sa valeur modale est neutre. Le plus souvent, il s'agit de la modalité caractéristique de l'indicatif, de la modalité factive. (1991:204) Y añade en nota: Il arrive que le participe exprime le 'temps absolu'. Lorsqu'il exprime le 'temps relatif', il arrive souvent qu'il adopte la modalité du verbe principal. Y a propósito del infinitivo dependiente de verbos de voluntad, deber, capacidad, etc (inf. dinámico) afirma: au point du vue temporel, l'infinitif exprime en principe que l'action es postérieure à l'action exprimée par le verbe dont il dépend; il s'agit donc de temps relatif (1991:204). Ahora atribuye al infinitivo una noción que descansa en el valor semántico del lexema verbal (cf. MARTINEZ VÁZQUEZ
} 
2) No hay una definición coherente del valor de las desinencias primarias y secundarias y falta una explicación de su relación con el sistema de temas verbales. A propósito del indicativo primario se insinúa ingeniosamente que el valor de la desinencia primaria sería el de identificar el punto de referencia (moment donné) como el momento presente:

L'indicatif primaire exprime que l'action ou l'état exprimé par le thème temporel comporte le moment présent. Le moment donné est ici le moment présent (...) Au point du vue morphologique, le grec constitue l'ind. prim. en ajoutant l'une des désinences primaires immédiatement après le thème temporel. (1991:201)

Parece introducir aquí la idea de que la desinencia temporal (primaria / secundaria) determina el punto de referencia, estableciendo una jerarquización de las categorías de tiempo relativo y tiempo absoluto semejante a la que hallamos en las teorías de la temporalidad de BELLO, seguido en nuestros tiempos por BULL, COMRIE y algún otro. Pero el valor de las desinencias vuelve a quedar en el aire cuando se define el valor del indicativo secundario:

L'indicatif secondaire exprime que l'action ou l'état exprimé par le thème temporel est situé dans le passé. (...) Pour l'indicatif secondaire du thème du présent (l'imparfait) et celui du thème du parfait (le plus-que-parfait), le moment donné est un moment du passé. (...) Pour l'ind. sec. du thème de l'aoriste, en revanche, le moment donné est soit le moment présent soit un moment du passé. Le premier cas concerne la simple constatation d'une action isolée du passé, emploi qui se présente notament dans les textes de conversation: हैா narratifs où il s'agit en principe d'une série d'actions cohérente: type 'il entra ..., il but du vin ..., (puis) il parla à son voisin ...'. Ici, l'aoriste ě $\pi \iota \epsilon$ 'il but' exprime que l'achèvement de l'action de boire était antérieur à l'action de parler au voisin. Pour

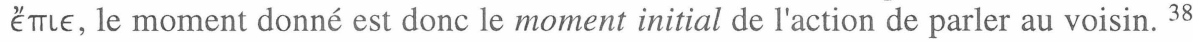
$(1991: 201)$

Ahora la desinencia (secundaria) sencillamente sitúa la acción verbal en el pasado, permitiendo una definición del indicativo de aoristo que evita toda referencia a valores aspectuales: como anterior de presente o anterior de pasado. Es difícil estar de acuerdo con esto.

3) Por otra parte, RUIJGH prescinde del término aspecto, porque prefiere limitar su empleo a la descripción del verbo eslavo. Pero juntamente con el término prescinde de la idea de que los temas verbales griegos pueden expresar determinaciones absolutas, no relativas a ningún momento concreto. Ello es consecuencia lógica de su tesis central, en que postula una sola categoría de índole temporal. Lo cual lo lleva a dar explicaciones en algunos casos un tanto forzadas. Valga de ejemplo la explicación de los empleos de los temas aspectuales de presente y de aoristo en el imperativo: 
Le choix entre l'impératif du présent et celui de l'aoriste dépend du moment donné. Lorsque le locuteur dit $\gamma \rho \alpha \dot{\phi \epsilon}$ 'écris' [impér. pres.], il a à l'esprit un moment futur

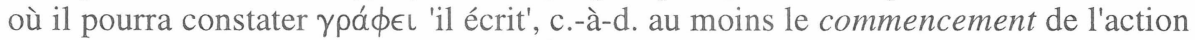
désirée. Lorsqu'il dit ypáłov 'écris' [impér.aor.], il a à l'esprit un moment futur où il pourra constater Ë $\gamma \rho a \psi \epsilon$ 'il a écrit', c.-à-d. l'achevement de l'action désirée. $(1991: 202)$

A RUIJGH, que da la impresión de seguir fielmente al Díscolo ${ }^{39}$, le acontece lo mismo que al gramático griego. En su empeño por explicar valores temporales, ambos se ven traicionados por el propio reconocimiento de valores aspectuales. Aquí RUIJGH viene a sostener que con el imperativo de presente se ordena el comienzo de la acción y con el imperativo de aoristo se ordena el acabamiento de ésta. Es el reconocimiento de un valor aspectual inceptivo o incoativo (variante del imperfectivo), en el presente, y perfectivo, en el aoristo, que se entienden bien desde la perspectiva de la acción en sí misma, sin recurrir de manera forzada a un momento ajeno a la acción, del que no hay constancia desde el punto de vista lingüístico.

\subsubsection{TEORÍA DEL ASPECTO RELATIVO.}

STAHL aporta una explicación alternativa a la de la transformación de mensaje directo en mensaje indirecto. Afirma que en las subordinadas adverbiales y de relativo ${ }^{40}$, así como participios adverbiales, en las que se puede apreciar un matiz de tiempo relativo (relative Zeitstufe), las formas del tema de presente y aoristo (subjuntivo ${ }^{41}$, optativo ${ }^{42}$ y participio ${ }^{43}$ ) expresan respectivamente simultaneidad y anterioridad como desarrollo secundario de su valor aspectual. El presente es la forma adecuada para expresar lo simultáneo, en la medida en que se considera como lo que dura junto a otro; para el pasado relativo o anterioridad en sí, dado que excluye la simultaneidad y la duración, el aoristo (1907:165).

En la misma línea parece sugerir KURYLOWICZ ${ }^{44}$ la hipótesis ${ }^{45}$ siguiente: los valores de anterioridad y simultaneidad serían producto de una realización de los valores aspectuales de

\footnotetext{
39 Cf. MARTÍNEZ VÁZQUEZ 1987.

40 Sólo las synthetische Nebensätze, es decir, excluidas las parathetische $N$., que incluyen casos de "falsa subordinación" o coordinación por subordinación y subordinación inversa. Excluye además, las temporales de posterioridad con $\ddot{E} \omega S$, $\pi \rho i v$ y conjunciones relacionadas. Cf. loc. cit.

41 1907:167 ss.

42 1907:173 ss.

43 1907:209 ss., 211 ss.

44 Este autor no acepta que el griego exprese nociones de anterioridad (anteriority), alegando, tras WACKERNAGEL (1950:151ss.), que en esta lengua, como en polaco, la anterioridad es una variante contextual independiente del aspecto perfectivo o imperfectivo de la forma verbal (1964:90). Sin embargo, en aquellas lenguas en que sí existe el tiempo relativo (=relative aspect), como el latín o el inglés, dicho autor lo explica a partir del siguiente razonamiento: el diagrama de oposiciones aspectuales (indeterminado / perfectivo / imperfectivo / estado) puede ser referido al momento de habla, a un momento pasado o a un momento futuro; entonces se tiene la categoría de "aspecto relativo" (categoría de anterioridad), característica de estas lenguas. Encuentro en esta tesis la misma dificultad de planteamiento ya citado antes. La formulación de la categoría de "aspecto relativo" como una categoría de anterioridad vs. simultaneidad (1964:90) deja fuera la noción de posterioridad patente en la taxis del latín o del español y en éstas lenguas no se emplean temas aspectuales para expresar esta noción de posterioridad, por lo que no se puede explicar globalmente esta categoría a partir de la
} 
perfectividad e imperfectividad en determinados contextos de dependencia sintáctica. De hecho, este autor propone sustituir la expresión tiempo relativo por otra de aspecto relativo: aspecto genuino y relación temporal ("aspecto relativo") no son incompatibles entre sí. La relación temporal puede ser expresada en lenguas con aspecto genuino (absoluto) y viceversa. Como regla solemos tratar con funciones secundarias, condicionadas por el contexto (1964:27).

Esta línea explicativa tiene interés para la vertiente genética del asunto. Es evidente que simultaneidad y anterioridad han surgido en griego como desarrollos de categorías aspectuales, de perfectividad e imperfectividad respectivamente. También la posterioridad aparece surgida a partir de la noción de tiempo futuro, que a su vez surgió de una modalidad desiderativa. Pero esta explicación ha servido en ocasiones ${ }^{46}$ más para enmascarar que para iluminar el hecho de que el griego expresa sistemáticamente el tiempo relativo, cada vez que lo necesita.

A ello contribuye el concepto de realización o de función secundaria dependiente del contexto. Es una visión parcial, miope del asunto. En la medida que ambos valores, de aspecto y de tiempo relativo o de tiempo absoluto y de tiempo relativo aparecen en distribución complementaria, cada uno de ellos, tanto el primario como el secundario, está condicionado por el contexto en que se realiza. La diferencia entre primario y secundario es bien genética bien basada en que un contexto es más fácilmente precisable que otro. Pero desde un punto de vista descriptivo es irrelevante: lo relevante es la delimitación de cada uno de los contextos.

4. EXPLICACIÓN DEL TIEMPO RELATIVO EN GRIEGO ANTIGUO. Las tres líneas explicativas reseñadas aportan luz sobre vertientes distintas del fenómeno de la expresión de tiempo relativo en griego antiguo. Pero tan sólo apuntan parcialmente en la dirección adecuada: la necesidad de describir los temas de aoristo, presente y futuro como formas con dos valores gramaticales como operadores de aspecto / tiempo relativo o tiempo absoluto / tiempo relativo en distribuciones complementarias. El problema queda así resuelto en dos niveles, el del significado y el de la distribución: determinadas categorías morfológicas (Pres. Aor. Fut.) muestran valores semánticos distintos en diferentes ámbitos o contextos de realización.

\footnotetext{
categoría de aspecto. Para obviar esta dificultad, parece que este autor propone un análisis de la noción de posterioridad aparte de las nociones de anterioridad / no anterioridad, en consecuencia, aparte de la categoría de anteriority. Así, el "condicional" del verbo inglés es analizado como término complejo de la categoría de tiempo (tense) (p.25): presente $(\Gamma=$ neutro / futuro $(B=$ negativo) / pasado $(\beta=$ positivo) / "condicional" (futuro de pasado) $(\gamma=$ complejo). Desde una perspectiva formal, el análisis parece adecuado al verbo inglés: I do /I will do / I did / I would do, donde I would do se muestra como una formación de "pasado del futuro". Pero este análisis no casaría con el verbo griego, donde sería recomendable, con RUIJGH, analizar el futuro junto a los demás temas verbales. Desde una perspectiva nocional, en cambio, no se entiende con qué criterio se separan las nociones de anterioridad y simultaneidad, asimismo complejas (pasado de pasado, ..., presente de pasado, ...), de la noción de posterioridad.

${ }_{45}$ Propuesta por mí en Aspecto y tiempo en la lengua de Polibio, Tesis Doctoral inédita, Sevilla, 1987.

46 Cf. ADRADOS y el propio KURYLOWICZ, más arriba.
} 
La solución ha de confirmarse aún en otro nivel, nivel funcional, de uso: el de las relaciones opositivas paradigmáticas de estos signos, según muestra la conmutación. Consideremos los ejemplos siguientes de infinitivos en su distribución completa:

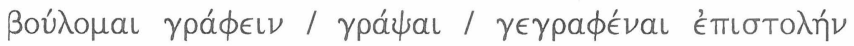

«deseo escribir (estar, seguir escribiendo) / escribir (acabar de escribir) / tener escrita una carta»

Tomemos el aoristo $\gamma \rho \alpha ́ \psi a \iota$. En esta distribución concreta (infinitivo dinámico) es conmutable por presente o por perfecto ${ }^{47}$ a los que se opone expresando nociones aspectuales. Observemos ahora los ejemplos de la otra distribución, es decir, los ejemplos de infinitivos "declarativos":

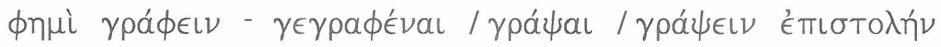

«afirmo que escribo (estoy escribiendo) - tengo escrita / he escrito / voy a escribir una carta»

Aquí el aoristo ypáłaı es conmutable por presente o perfecto y ipor futuro! Evidentemente las nociones no son las mismas: presente y perfecto expresan simultaneidad de la acción o del estado resultante; aoristo, anterioridad; futuro, posterioridad. Pero tampoco lo son las relaciones opositivas: el aoristo ahora se opone al bloque presente - perfecto y al futuro. $\mathrm{Y}$ si existe oposición, como muestra la conmutación, es que los términos pertenecen a la misma categoría.

${ }^{47}$ El futuro es excepcional en dependencia de verbos como éste. STAHL (1907:202-4) propone corregir los textos y es seguido por varios editores. CLASSEN ( $a d$ Th. 6.6.1) es partidario de mantenerlos. 


\section{REFERENCIAS BIBLIOGRÁFICAS}

ADRADOS, F.R.

COMRIE, B.

CRESPO, E.

HETTRICH, $\mathrm{H}$.

HOLT, J.

HUMBERT, J.

JACOBSON, R.O.

KURYLOWICZ, J.

KÜHNER, R. - GERTH, B.

MARTÍNEZ VÁZQUEZ, R.

OGUSSE, A.

RIJKSBARON, A.

RUIJGH, C.J.

SCHWYZER, E. -

DEBRUNNER, A.

SMYTH, H.W.

STAHL, J.

STORK, P.

WACKERNAGEL, J.
(1992) Nueva Sintaxis del Griego Antiguo (Madrid: Gredos 1992).

(1976) Aspect (Cambridge:C.U.P 1976).

(1984) "Infinitivo modal sin åv en griego", EC 87 (1984) 6773.

(1976) Kontext und Aspekt in der altgriechischen Prosa Herodots, (Göttingen:Vandenhoek \& Ruprecht 1976).

(1942) Etudes d' Aspect (Copenhagen:Munskgaard 1943).

(1960) Syntaxe Grecque (Paris:Klincksieck 1960).

(1984) "Los conmutadores, las categorías verbales y el verbo ruso", en Ensayos de lingüística general (Barcelona:Ariel 1984) 307-332.

(1964) The Inflectional Categories of Indo-European (Heidelberg:C.Winter 1964).

(1898) Ausführlische Grammatik der griechischen Sprache II.1 (Hannover:Hahnsche Buchhandlung 1966 [1898]).

(1904) Ausführlische Grammatik der griechischen Sprache II.2 (Hannover:Hahnsche Buchhandlung 1966 [1904]).

(1987) "Aspecto verbal en Apolonio Díscolo: el tema de aoristo", Actas del VII Congreso Español de Estudios Clásicos (Madrid 1989 [1987]).

(1989) "Infinitivos dinámicos e infinitivos declarativos en griego antiguo", Emerita 57 (1989) 293-307.

(1962) Recherches sur le participe circonstanciel en grec ancien (Strasbourg 1962).

(1984) The syntax and Semantics of the Verb in Classical Greek (Amsterdam:Gieben 1984).

(1971) Autour de "TE épique" (Amsterdam:Hakkert 1971).

(1991) "Les valeurs temporelles des formes verbales en grec ancien", en The Function of Tense in Texts, ed. J. GVOZDANOVIC (Amsterdam:North Holland 1991, 197-217.

(1969) Griechische Grammatik. II, Syntax und syntaktische Stylistik: (München 1949 [reimp. 1969]).

(1963) Greek Grammar (Cambridge:Harvard University Press 1963 [1920]).

(1907) Kritisch-historische Syntax des griechischen Verbums der klassischen Zeit (Hildesheim:Georg Olms 1965 [1907]). (1982) The Aspectual Usage of the Dynamic Infinitive in Herodotus (Groningen:Bouma's Boekhuis 1982).

(1950) Vorlesungen über Syntax I, (Basel:Emil Birkhäuser [1928]). 
